Conaghan PG, Panayi GS, Klareskog L, Emery P, Nissim A. Autoantibodies to posttranslationally modified type II collagen as potential biomarkers for rheumatoid arthritis. Arthritis Rheum 2013 July;65(7):1702-12.

Disclosure of Interest: None declared DOI: 10.1136/annrheumdis-2018-eular.4404

\section{FRI0160 INTERLEUKIN-17A INDUCES INFLAMMATORY RESPONSE VIA NLRP3 INFLAMMASOME IN ANKYLOSING SPONDYLITIS}

D. Kim, S.-W. Lee, J.J. Song, Y.-B. Park, M.-C. Park. Division of Rheumatology, Yonsei University College of Medicine, Seoul, Korea, Republic Of

Background: Inflammasomes are cytoplasmic multiprotein complexes that recognise various exogenous and endogenous danger signals in myeloid cells, particularly macrophages. Inflammasome activation eventually induces inflammatory responses in macrophages by activating gasdermin-d-mediated pyroptosis and the secretion of pro-inflammatory cytokines including interleukin (IL)-1 $\beta$ and IL18 in a caspase-1-dependent manner. The production of IL-1 has been found to be highly induced in $\mathrm{AS}^{1}$ and caspase-1 level was significantly elevated in spondyloarthritis patients than in those with other arthritic diseases, ${ }^{2}$ suggesting the possibility that inflammasomes might be involved in pathogenesis of ankylosing spondylitis (AS). However, few studies have addressedd the roles of inflammasomes in AS.

Objectives: This study was performed to investigate the role of NOD-like receptor family, pyrin domain containing 3 (NLRP3) inflammasome in the peripheral blood mononuclear cells (PBMCs) from AS patients.

Methods: PBMCs from 11 patients and 9 healthy controls were isolated and mRNA expression levels of NLRP3 inflammasome, pro-IL-1 $\beta$, IL-1 $\beta$, pro-caspase-1, and caspase-1 were determined using quantitative real-time PCR. IL-17A (100 ng/mL) was added to the cell cultures, then, cytokine expression, signalling pathways, and inflammasome machinery are determined using real-time RT-PCR and Western blot. After transfection of siNLRP3 to AS PBMCs, mRNA and proteins levels of IL-1 $\beta$ and caspase- 1 were determined using real time PCR and western blot.

Results: Expressions of NLRP3, IL-1 1 , and caspase- 1 mRNAs, but not IL-18, were increased in PBMCs from AS patients compared to controls. Incubation of AS PBMCs with IL-17A significantly increased NLRP3, IL-1 $\beta$, IL-18, and caspase1 expressions in AS PBMCs. Western blot showed IL-17A treatment induced the phosphorylation of Akt, p38 MAPK, and NF-kB p65 in AS PBMCs. Down-regulation of NLRP3 by transfection of siRNA decreased mRNA expressions and productions of IL-1 $\beta$ and caspase- 1 in AS PBMCs.

Conclusions: Activity of NLRP3 inflammasome was increased in AS patients and IL-17A potentiated the activation of NLRP3 inflammasome. Our data suggest the possible role of NLRP3 inflammasome in inflammatory response in AS.

\section{REFERENCES:}

[1] Tan AL, Marzo-Ortega H, O'Connor P, Fraser A, Emery P, McGonagle D. Efficacy of anakinra in active ankylosing spondylitis: a clinical and magnetic resonance imaging study. Ann Rheum Dis. 2004;63:1041-1045.

[2] Son CN, Bang SY, Kim JH, Choi CB, Kim TH, Jun JB. Caspase-1 level in synovial fluid is high in patients with spondyloarthropathy but not in patients with gout. J Korean Med Sci. 2013;28:1289-1292.

Disclosure of Interest: None declared

DOI: 10.1136/annrheumdis-2018-eular.3014

\section{FRI0161 ROLE OF GITR/GITRL IN MODULATING TH9 RESPONSE IN PSORIATIC ARTHRITIS}

G. Guggino ${ }^{1}$, D. di liberto ${ }^{1}$, A. Rizzo ${ }^{2}$, M. La Manna ${ }^{1}$, A. Ferrante ${ }^{1}$, L. saieva ${ }^{1}$, R. Alessandro', G. Sireci ${ }^{1}$, F. ciccia ${ }^{1}$. ${ }^{1}$ Università Degli Studi Palermo; ${ }^{2}$ Ospedali riuniti Villa Sofia-Cervello, Palermo, Italy

Background: GITR/GITRL interaction is pivotal in regulating the activity of Treg and of Th9 effector cells. Psoriatic arthritis (PsA) is a chronic inflammatory disease characterised by a strong expression of IL-9 and Th9 polarisation in the inflamed gut, synovial tissues and peripheral blood. ${ }^{2}$ Factors involved in the regulation of Th9 responses in PsA have been not yet investigated.

Objectives: Aim of the study: to investigate the role of GITR/GITRL in PsA

Methods: GITR, GITRL expression were assessed by rt-PCR and immunohistochemistry in synovial biopsies, gut specimens and bone marrow of PsA patients and healthy controls (HC). The co-localization of CD4 and GITR was analysed by immunofluorescence. The expression of GITR was also assessed by flow cytometry analysis in isolated PBMC of patients and controls (Tregs, CD4 and CD8).
Peripheral and intestinal IL-9 producing cell (Th9) percentages and cytotoxic activity of T lymphocytes were also studied by flow cytometry analysis ex vivo and after in vitro stimulation with GITRL. A mouse model of induced arthritis (collagen induced arthritis, CIA) was used to study GITR/GITRL axis and the effect of antiGITRL blocking agent.

Results: Increased GITR and GITRL expression was observed in the inflamed gut and synovial samples of PsA patients. Analysis of GITR expression among PBMC and LPMC from PsA patients demonstrated its down-regulation among Tregs and upregulation on effector CD4 +and CD8+T cells. In in vitro studies, GITR co-stimulation potently induced Th9 activation and IL-9 production. In particular, GITR ligation subverted the induction of Foxp3(+) Tregs, directing the activated CD4(+) T cells to a Th9 phenotype and enhancing the function of DCs and cytotoxic T lymphocytes. Moreover, in a murine model of CIA massive expression of IL- 9 and GITR was observed in the synovial tissues and anti-GITR therapy sig nificantly ameliorated arthritis score.

Conclusions: We demonstrated that GITR/GITRL axis modulates IL-9/Th9 responses in PsA, representing GITR activation a relevant upstream pathway involved in Th9 polarisation. A novel mechanism by which GITR agonist exert an inflammatory response was also demonstrated in PsA indicating GITR blocking agents as possible therapy in PsA.

\section{REFERENCES :}

[1] Xiao X, Shi X, Fan Y, Zhang X, Wu M, Lan P, Minze L, Fu YX, Ghobrial RM, Liu W, Li XC. GITR subverts Foxp3(+) Tregs to boost Th9 immunity through regulation of histone acetylation. Nat Commun. 2015 Sep $14 ; 6: 8266$.

[2] Ciccia F, Guggino G, Ferrante A, Raimondo S, Bignone R, Rodolico V, Peralta S, Van Tok M, Cannizzaro A, Schinocca C, Ruscitti P, Cipriani P, Giacomelli R, Alessandro R, Dieli F, Rizzo A, Baeten D, Triolo G. Interleukin-9 Overexpression and Th9 Polarization Characterize the Inflamed Gut the Synovial Tissue, and the Peripheral Blood of Patients With Psoriatic Arthritis. Arthritis Rheumatol 2016 Aug;68(8):1922-31.

[3] Xiao X, et al. GITR subverts Foxp3(+) Tregs to boost Th9 immunity through regulation of histone acetylation. Nat Commun. 2015 Sep.

[4] Ciccia F, Guggino G, et al. Interleukin-9 Overexpression and Th9 Polarization Characterize the Inflamed Gut, the Synovial Tissue, and the Peripheral Blood of Patients With Psoriatic Arthritis. Arthritis Rheumatol 2016 Aug.

Disclosure of Interest: None declared

DOI: 10.1136/annrheumdis-2018-eular.6449

\section{FRI0162 THE LINK BETWEEN ANGIOGENESIS AND OSTEOGENESIS IN SPONDYLOARTHRITIS}

M.H. Kaaii ${ }^{1,2}$, J.P. van Hamburg ${ }^{1,2}$, G. Kollias ${ }^{3,4}$, D.L.P. Baeten ${ }^{1,2}$, L.M. van Duivenvoorde ${ }^{1,2}$, S.W. Tas ${ }^{1,2} .{ }^{1}$ Amsterdam Rheumatology and immunology Center, ${ }^{2}$ Department of Experimental Immunology, AMC/University of Amsterdam, Amsterdam, Netherlands; ${ }^{3}$ Division of Immunology, Biomedical Sciences Research Center Alexander Fleming, Vari; ${ }^{4}$ Department of Physiology, Medical School, National and Kapodistrian University of Athens, Athens, Greece

Background: Spondyloarthritis is characterised by inflammation, extensive angiogenesis and pathological osteogenesis. Transmembrane (tm)TNF trans genic (tg) mice that overexpress tmTNF exhibit features of $\mathrm{SpA}$, including chronic inflammation and pathological osteogenesis. tmTNF ligation to TNF receptor 2 in endothelial cells (ECs) can induce signal transduction pathways, that may promote these processes. Of note, angiogenesis and osteogenesis are coupled by EC differentiation towards a type $\mathrm{H}$ (CD31 ${ }^{\text {hi }}$ endomucin $\left.{ }^{\text {hi }}\right)$ phenotype. ${ }^{2}$

Objectives: To investigate the link between pathological angiogenesis and osteogenesis in tmTNF tg mice.

Methods: Vertebrae and ankles from 6 and 12 weeks and 8 months old tmTNF tg mice or sex-matched non-tg littermates $(n=18)$ were prepared by cutting $60 \mu \mathrm{m}$ thick cryosections for confocal imaging.

Results: tmTNF tg mice exhibited ectopic osteogenesis which was not observed in non-tg littermates. The provided image demonstrates an ectopic lesion at the white arrow. Immunostainings showed that type $\mathrm{H}$ vessels are in the vicinity of the ectopic osteogenesis and osterix ${ }^{+}$osteoprogenitors. At six weeks of age, osterix ${ }^{+}$ cells are located throughout the ectopic lesion, while at eight months, osterix ${ }^{+}$cells are only present at the border of the lesion. Furthermore, there is increased osteogenesis and a different vessel architecture within the vertebrae of tmTNF tg mice compared to non-tg littermates that progresses with age. Non-tg littermate vertebrae only have physiological osteogenesis, which is in the metaphysis and perios teum. In addition, tmTNF tg mice also exhibit altered bone marrow (BM) architecture containing extensive lymphoid aggregates, which predominantly consisted of $\mathrm{B} 220^{+} \mathrm{B}$ cells. 


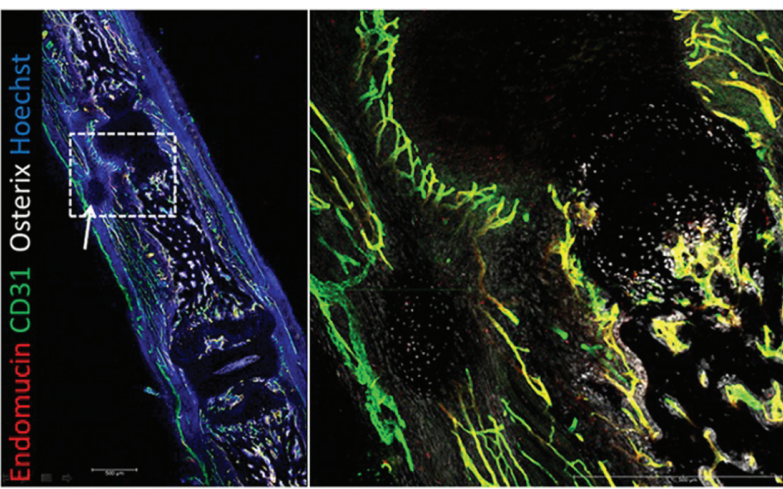

Abstract FRI0162 - Figure 1. Type $\mathrm{H}$ vessel association with ectopic bone formation in 6week-old murine vertebra. Left panel: Confocal tile scan of tmTNF tg vertebrae showing endomucin* ${ }^{*}$ (red), which labels all vessels except arteries, and CD $31^{*}$ (green) endothelia cells. Osterix (white) labels osteoprogenitors and nuclei are labelled by Hoechst (blue). Right panel: Higher magnification of osterix* osteoprogenitors at ectopic location. Osterix* cells are found around type $\mathrm{H}\left(\mathrm{CD} 31^{\text {hi }}{ }^{\text {endomucin }}{ }^{\text {hi }}\right)$ vessels.

Conclusions: tmTNF overexpression in mice leads to development of type $\mathrm{H}$ vessels associated with ectopic osteogenesis. In addition, extensive lymphoid aggregates develop in the BM. Current studies are aimed at identification of signalling pathways in ECs that contribute to these processes.

\section{REFERENCES:}

[1] Alexopoulou L, et al. Eur J Immunol 1997;27(10):2588-92.

[2] Kusumbe AP, et al. Nature 2014;507(7492):323-328.

Disclosure of Interest: None declared

DOI: 10.1136/annrheumdis-2018-eular.4419

\section{FRI0163 DEVELOPMENT OF A PRECLINICAL TESTING PIPELINE FOR A NOVEL TRANSMEMBRANE TNF-DRIVEN SPONDYLOARTHRITIS MODEL}

K. Kranidioti ${ }^{1}$, L. Ntari ${ }^{1}$, E. Argyropoulou ${ }^{1}$, C. Geka ${ }^{1}$, N. Karagianni ${ }^{1}$, M.C. Denis ${ }^{1}$, G. Kollias ${ }^{2,3} .{ }^{1}$ Biomedcode Hellas SA; ${ }^{2}$ Institute of Immunology, Biomedical Sciences Research Center (BSRC) Alexander Fleming, Vari; ${ }^{3}$ Department of Physiology, School of Medicine, National and Kapodistrian University of Athens, Athens, Greece

Background: Spondyloarthritis (SpA) is a complex disease characterised by chronic inflammation, bone erosion and pathological new bone formation. The TgA86 transmembrane TNF (tmTNF) transgenic mouse is a unique model of SpA, developing spontaneously and with $100 \%$ incidence early progressive SpA characterised by peripheral inflammatory arthritis and axial ankylosing spondylitis with cardiovascular involvement. This closely recapitulates the pathological findings and comorbid conditions described in human patients.

Objectives: To characterise in greater detail the development and progression of theTgA86 pathology and its similarities to human disease and to standardise reliable preclinical protocols and specific readouts for the assessment of the efficacy of human therapeutics.

Methods: TgA86 peripheral and axial pathology was assessed at different time points from 2.5 to 28 weeks of age. Disease severity was evaluated using clinical parameters and histopathological analysis of ankle and sacroiliac joints, lumbar and caudal vertebrae, as well as whole mount skeletal staining. Clinical and histopathological readouts were used to assess the therapeutic effect of Etanercept that was administered thrice weekly at $30 \mathrm{mg} / \mathrm{Kg}$ starting either from $2.5-5$ weeks of age (prophylactic protocol) or from 9 weeks of age (therapeutic protocol).

Results: Clinical pathology in TgA86 mice appears already from 2.5 weeks of age, with signs of paw swelling, digit deformation and tail crinkling, while by 9 weeks of age pathology is fully established, with severe peripheral arthritis and tail and spine ankylosis. Pathology progression was also evident histopathologically, characterised by the originally described features of progressive inflammation, cartilage destruction and bone erosion observed in sacroiliac and ankle joints as well as in lumbar and caudal vertebra. Additionally, new pathology features were detected by identifying signs of enthesitis, new bone formation appearing as cartilaginous structures at the edges of vertebrae endplates, presence of red bone marrow during all stages of disease progression as well as signs of intervertebral disc (IVD) degeneration. Prophylactic treatment with Etanercept ameliorated effectively all clinical and histopathological features of the peripheral and axial pathology. Therapeutic treatment while affecting only minimally the clinical signs of both peripheral and axial pathology, it was found to reduce the periphera arthritis histopathological score by at least $50 \%$. Finally, treatment with Etanercept was also efficient in ameliorating the comorbid heart valve pathology observed in these animals.

Conclusions: We have shown that TgA86 pathology includes features of sacroiliitis, enthesitis, new bone formation, persisting red bone marrow and intervertebral disk degeneration, further strengthening the similarities of this model to human pathology. Based on the assessment of all pathology features during prophylactic anti-TNF treatment we suggest that early on in disease there may be a therapeutic window during which optimal treatment of the pathology can be achieved.

\section{REFERENCE:}

[1] Alexopoulou L, Pasparakis M, Kollias G. A murine transmembrane tumor necrosis factor (TNF) transgene induces arthritis by cooperative p55/p75 TNF receptor signaling. Eur J Immunol 1997;27(10):2588-92.

Disclosure of Interest: None declared

DOI: 10.1136/annrheumdis-2018-eular.5508

\section{FRI0164 ANTI-PFDN5 ANTIBODY AS A BIOMARKER FOR UVEITIS IN ANKYLOSING SPONDYLITIS}

O.C. Kwon ${ }^{1}$, E.-J. Lee ${ }^{1}$, E.-J. Chang ${ }^{2}$, W.J. Seo ${ }^{3}$, J.S. Oh ${ }^{4}$, S. Hong ${ }^{1}$, C.-K. Lee ${ }^{1}$, B. Yoo ${ }^{1}$, Y.-G. Kim ${ }^{1}$. ${ }^{1}$ Division of Rheumatology, Department of Medicine, University of Ulsan, College of Medicine, Asan Medical Center, ${ }^{2}$ Department of Biomedical Sciences, University of Ulsan College of Medicine, Asan Medical Center, ${ }^{3}$ Division of Rheumatology, Department of Medicine, Seoul Veterans Hospital; ${ }^{4}$ Clinical Research Center, University of Ulsan College of Medicine, Asan Medical Center, Seoul, Korea, Republic Of

Background: Noninfectious uveitis is the most common extra-articular manifestation of ankylosing spondylitis (AS). However, molecules related to the disease pathogenesis have yet to be identified, and no biomarkers are available for uveitis in AS.

Objectives: We aimed to identify the biomarkers for uveitis in AS, and elucidate the possible pathogenesis of uveitis in AS associated with the identified biomarkers.

Methods: Protein microarray using ProtoArray was performed to profile autoantibodies present in sera from patients with various autoimmune diseases, including eight AS patients with uveitis. The autoantibodies with higher reactivity in AS patients with uveitis compared with other patients were selected, and the levels of autoantibodies were measured using ELISA in the sera from AS patients with $(n=32)$ or without uveitis history $(n=32)$, patients with rheumatoid arthritis $(n=20)$ and from healthy individuals $(n=12)$. To evaluate the involvement of target antigen in pathogenesis of spondyloarthritis-related uveitis, we conducted an in vivo study using curdlan-induced SKG mice, which spontaneously develops arthritis as well as uveitis, and an in vitro study using retinal pigment epithelium cell-line (RPE19). Results: 4 antibodies (Abs) were selected as a candidate for biomarker (antiPFDN5, area under curve $[A \cup C]=1.00$; anti-serine threonine protein kinase $24 \mathrm{Ab}$ $A \cup C=0.906$; anti-odontogenic ameloblast associated protein $A b, A \cup C=0.859$; anti-protocadherin alpha $\mathrm{C} 2 \mathrm{Ab}, \mathrm{AUC}=0.859)$. In ELISA, anti-PFDN5 Abs was sig nificantly elevated in AS patients with uveitis compared to AS patients without uveitis. Ocular histology showed that compared to PBS-treated SKG mice, SKG mice with uveitis had strong expression of PFDN5 in iris, ciliary body, and retina PERK and pelF2 $\alpha$, which are ER stress related proteins, were downregulated in PFDN5 siRNA-treated RPE19 cells in tunicamycin-induced condition, suggesting that PFDN5 enhances ER stress via PERK and pelF2 $\alpha$ pathway.

Conclusions: We identified anti-PFDN5 antibody as a putative biomarker for uveitis in AS. PFDN5 was increased in ocular lesion, which may be associated with disease pathogenesis.

Acknowledgements: None.

Disclosure of Interest: None declared

DOI: 10.1136/annrheumdis-2018-eular.4396 